\title{
Shifted Known Symbol Padding for Efficient Data Communication in a WLAN Context
}

\author{
Olivier Rousseaux • Geert Leus • Marc Moonen
}

Published online: 22 September 2007

(C) Springer Science+Business Media, LLC 2007

\begin{abstract}
To allow for a computationally efficient equalization scheme for the frequencyselective transmission channels encountered in wireless local area network (WLAN) applications, cyclic prefix (CP) block transmission schemes have been proposed, such as single-carrier $\mathrm{CP}$ (SC-CP) and multi-carrier CP (MC-CP) transmission, also known as orthogonal frequency division multiplexing (OFDM). In this letter, however, we focus on the known symbol padding (KSP) transmission scheme. In this scheme known padded sequences can be exploited for synchronization as well as for channel estimation. However, to simultaneously allow for low-complexity frequency-domain equalization and accurate channel estimation within the KSP context, a modified KSP scheme is proposed, namely shifted KSP (S-KSP). Comparing different block transmission schemes in the WLAN context, the S-KSP scheme is shown to offer a very good performance.
\end{abstract}

Keywords Wireless communications - Wireless local area networks · OFDM · Block transmission · Diversity techniques · Channel estimation

\section{Introduction}

To offer competitive data transmission speeds, wireless local area networks (WLANs) rely on broadband communication channels. Multipath effects, resulting in frequency-selective

\section{O. Rousseaux}

Holst Centre - IMEC NL, High Tech Campus 42, Eindhoven, The Netherlands, e-mail: olivier.rousseaux@imec-nl.nl

G. Leus $(\varangle)$

Faculty of EEMCS, Delft University of Technology, Mekelweg 4, Delft 2628 CD, The Netherlands e-mail: g.leus@tudelft.nl

M. Moonen

Department of ESAT, Katholieke Universiteit Leuven, Kasteelpark Arenberg 10, Heverlee B-3001, Belgium

e-mail: marc.moonen@esat.kuleuven.ac.be 
fading, are then a major impediment of broadband communication systems. Frequencyselective fading introduces inter symbol interference (ISI), which needs to be tackled by appropriate equalization techniques at the receiver. Classical serial linear equalization schemes are known to offer sub-optimal performance. In this context, block transmission techniques based on the use of a cyclic prefix $(C P)$ have attracted a lot of attention in the last years for they allow for an efficient and computationally cheap ISI cancellation procedure [1-3]. Transmission schemes based on a CP can be classified into single-carrier cyclic prefix (SC-CP) transmission and multi-carrier cyclic prefix (MC-CP) transmission, also known as orthogonal frequency division multiplexing (OFDM). CP-based schemes allow for one-tap frequency-domain equalization. This has a very limited computational complexity whilst it effectively cancels the effects of the multipath transmission channel.

In this letter, we focus on the known symbol padding (KSP) scheme [4], in which a sequence of known symbols is padded to every block of transmitted data symbols. These padded sequences can be exploited for time and frequency synchronization [4-6], channel estimation [7, 8] or direct equalizer design [9]. However, KSP does not simultaneously allow for low-complexity frequency-domain equalization and accurate channel estimation. To solve this problem, we propose a modified KSP scheme, labeled shifted KSP (S-KSP), which allows for a coupling of both advantages.

\section{Known Symbol Padding}

Let us consider a communication link characterized by a stationary finite impulse response (FIR) channel of order $L$, denoted by $h[l]$, i.e., $h[l]=0$ for $l<0$ and $l>L$. If a sequence $x[n]$ is transmitted over this channel, the received sequence $y[n]$ is the linear convolution of the transmitted sequence with the channel impulse response:

$$
y[n]=\sum_{l=0}^{L} h[l] x[n-l]+\eta[n],
$$

where $\eta[n]$ is the additive white Gaussian noise (AWGN) at the receiver. In block transmission techniques, the data symbols are organized in blocks and fed through a precoding matrix before transmission. In addition, training symbols can be mixed with data symbols, leading to affine precoding [10]. Popular examples of block transmission schemes are single-carrier cyclic prefix (SC-CP) and multi-carrier cyclic prefix (MC-CP) transmission, also known as orthogonal frequency division multiplexing (OFDM). They both allow for low-complexity frequency-domain equalization (see [1-3] for more details).

Here, we discuss another type of block transmission, referred to as known symbol padding (KSP) [4]. With $k=0,1, \ldots, K-1$ representing the block index, a block $\mathbf{s}_{k}\left(N_{s} \times 1\right)$ of data symbols is defined as $\mathbf{s}_{k}=\left[s_{k}[0], \ldots, s_{k}\left[N_{s}-1\right]\right]^{T}$. A block $\mathbf{t}_{k}\left(N_{t} \times 1\right)$ of training symbols is similarly defined as $\mathbf{t}_{k}=\left[t_{k}[0], \ldots, t_{k}\left[N_{t}-1\right]\right]^{T}$. In a KSP transmission scheme, the $k$ th block of transmitted symbols, $\mathbf{x}_{k}:=\left[x\left[(k-1) N_{x}\right], \ldots, x\left[k N_{x}-1\right]\right]^{T}$, is then constructed as $\mathbf{x}_{k}=\left[\mathbf{s}_{k}^{T}, \mathbf{t}_{k}^{T}\right]^{T}$, i.e., $N_{x}=N_{s}+N_{t}$. The corresponding block of received symbols, $\mathbf{y}_{k}:=\left[y\left[(k-1) N_{x}\right], \ldots, y\left[k N_{x}-1\right]\right]^{T}$, can then be written as (assume $N_{t} \geq L$ )

$$
\mathbf{y}_{k}=\mathbf{H}_{K S P} \mathbf{s}_{k}+\mathbf{H}_{t 0} \mathbf{t}_{k}+\mathbf{H}_{t 1} \mathbf{t}_{k-1}+\boldsymbol{\eta}_{k},
$$

where $\boldsymbol{\eta}_{k}$ is similarly defined as $\mathbf{y}_{k}, \mathbf{H}_{K S P}$ is an $N_{x} \times N_{s}$ Toeplitz matrix with $[h[0], \ldots, h[L], 0, \ldots, 0]^{T}$ on the first column, $\mathbf{H}_{t 0}$ is an $N_{x} \times N_{t}$ lower triangular Toeplitz matrix with $[0, \ldots, 0, h[L], \ldots, h[0]]$ on the last row and $\mathbf{H}_{t 1}$ is an $N_{x} \times N_{t}$ upper triangular 
matrix with $[0, \ldots, 0, h[L], \ldots, h[1]]$ on the first row. Clearly, due to the fact that $N_{t} \geq L$, there is no interference between data blocks $\mathbf{s}_{k}$.

An important observation is that when the same training sequence is used for all data blocks, i.e., $\mathbf{t}_{k}=\mathbf{t}, \forall k$, the system can be described with a circulant channel matrix:

$$
\mathbf{y}_{k}=\left[\mathbf{H}_{K S P} \mid \mathbf{H}_{t 1}+\mathbf{H}_{t 0}\right]\left[\frac{\mathbf{s}_{k}}{\mathbf{t}}\right]+\eta_{k}=\mathbf{H}_{c i r c} \mathbf{x}_{k}+\eta_{k},
$$

where $\mathbf{H}_{\text {circ }}$ is an $N_{x} \times N_{x}$ circulant matrix with $[h[0], 0, \ldots, 0, h[L], \ldots, h[1]]$ on the first row. Describing the system with a circulant channel matrix is highly desirable as it allows for the use of low-complexity frequency-domain equalizers relying on the diagonalization properties of circulant matrices.

However, when the padded sequences are used for channel estimation purposes, the use of non-constant training sequences, i.e., $\mathbf{t}_{k} \neq \mathbf{t}_{l}, \forall k \neq l$, largely improves the quality of the channel estimates, irrespective of the estimation method that is used [8]. More specifically, the Cramer-Rao bound (CRB) analysis presented in [8] indicates that the channel modeling error tends to zero when there exists an exact solution to the channel estimation problem in the noiseless case. When no exact solution exists in the noiseless case, an error floor appears. When the number of transmitted blocks $K$ is sufficiently large, the use of non-constant training sequences yields an exact solution in the noiseless case as soon as $N_{t} \geq L+1$. When constant training sequences are used, this happens only when $N_{t} \geq 2 L+1$. But even when the channel order $L$ is sufficiently small to guarantee identifiability for non-constant as well as constant training sequences, the first always outperforms the latter [8].

In conclusion, constant training sequences allow for low-complexity frequency-domain equalization, while non-constant training sequences allow for accurate channel estimation. Both features can not be combined in traditional KSP.

\section{Shifted Known Symbol Padding}

In this section, we present a modified KSP scheme that offers the possibility of describing the transmission system with a circulant channel matrix whilst using non-constant training sequences, thereby allowing simultaneously for low-complexity frequency-domain equalization and accurate channel estimation.

Define the $\left(K+N_{t}-1\right) \times 1$ vector of known symbols $\boldsymbol{\tau}$. Define the $k$ th block of known symbols as $\mathbf{t}_{k}=\boldsymbol{\tau}\left(k: k+N_{t}-1\right)$. The requirement on $N_{t}$ is changed from the usual $N_{t} \geq L$ into $N_{t} \geq L+1$ and the block of received samples is now defined as $\mathbf{y}_{k}^{\prime}:=\mathbf{y}_{k}\left(1: N_{x}-1\right)=$ $\left[y\left[(k-1) N_{x}\right], \ldots, y\left[k N_{x}-2\right]\right]^{T}$, i.e., the last received symbol in $\mathbf{y}_{k}$ is simply discarded. Since the last $N_{t}-1$ symbols of $\mathbf{t}_{k-1}$ are equal to the first $N_{t}-1$ symbols of $\mathbf{t}_{k}$, the cyclicity of the channel input is restored. If $\mathbf{H}_{K S P}^{\prime}, \mathbf{H}_{c i r c}^{\prime}, \mathbf{H}_{t 1}^{\prime}$ and $\mathbf{H}_{t 2}^{\prime}$ are defined as the $\mathbf{H}_{K S P}, \mathbf{H}_{c i r c}$, $\mathbf{H}_{t 1}$ and $\mathbf{H}_{t 2}$ defined above, but then with modified dimensions $\left(N_{t}-1\right.$ is used instead of $N_{t}$ in the definitions), it is straightforward to check that $\mathbf{H}_{\text {circ }}^{\prime}=\left[\mathbf{H}_{K S P}^{\prime} \mid \mathbf{H}_{t 1}^{\prime}+\mathbf{H}_{t 2}^{\prime}\right]$. The transmission scheme can then be expressed as:

$$
\mathbf{y}_{k}^{\prime}=\mathbf{H}_{\text {circ }}^{\prime}\left[\frac{\mathbf{s}_{k}}{\mathbf{t}_{k}\left(1: N_{t}-1\right)}\right]+\eta_{k}^{\prime},
$$

where $\boldsymbol{\eta}_{k}^{\prime}$ is similarly defined as $\mathbf{y}_{k}^{\prime}$. Hence, at the cost of one extra redundant symbol, we have created the possibility of describing the transmission system with a circulant channel matrix whilst using non-constant training sequences. We refer to the scheme described here as shifted $K S P(S-K S P)$. 


\section{Simulation Results}

\subsection{Comparison of the Different Equalization Schemes}

In this section, we compare the performance of a number of transceiver schemes under the hypothesis that both the channel and the noise statistics are perfectly known at the receiver. We compare the SC-CP and OFDM schemes with the KSP scheme. For SC-CP and OFDM we consider linear minimum mean squared error (LMMSE) frequency-domain (FD) equalization. For KSP, we also consider LMMSE FD equalization but additionally investigate optimal LMMSE equalization, which is obtained from (2) by removing the influence of the training sequences and applying an LMMSE equalizer based on $\mathbf{H}_{K S P}$. Note that SC-CP and KSP with FD equalization have the same performance. Hence, we only show KSP with FD equalization.

We consider randomly generated additive white Gaussian noise of variance $\sigma^{2}$ : $\mathrm{E}\left\{\eta[i] \eta[j]^{*}\right\}=\delta_{i j} \sigma^{2}$. Different constellations are considered for the data symbols, i.e. BPSK, QPSK, 16-QAM and 64-QAM. These constellations are always scaled such that the variance of the data symbols is 1 , i.e., $\mathrm{E}\left\{s_{k}[i] s_{k}[i]^{*}\right\}=1$. The signal to noise ratio (SNR) is then defined as $\mathrm{SNR}=\mathrm{E}\left\{\|\mathbf{h}\|^{2}\right\} / \sigma^{2}$.

We simulate the performance in a context that is similar to the physical layer of the WLAN standard IEEE 802.11a, which relies on the OFDM transmission scheme. We simulate here how the overall system performance is changed when block transmission techniques other than OFDM are considered, while keeping the other parameters proposed in the standard (constellation mapping, error coding, data interleaving, block length, guard duration, ...) unchanged. IEEE 802.11a uses an OFDM transmission scheme with 64 subcarriers $\left(N_{s}=64\right)$ and a guard duration of 16 samples (or 8 in an optional mode). The data are transmitted in long bursts (typically several hundreds of OFDM symbols). Before transmission, the burst of binary data is encoded for error correction with a binary convolutional code. The coded data are organized in blocks, each block corresponding to a single OFDM symbol. A constellation-dependent interleaver operating on a per block basis is used in order to improve the efficiency of the convolutional code. Interleaved binary data are then mapped onto data symbols before OFDM transmission. A preamble used by the receiver for carrier synchronization and channel estimation is appended at the beginning of each transmitted burst. At the receiver, the data symbols are estimated with a classical OFDM equalizer and a demapper translates them into binary data that are then de-interleaved before going through a Viterbi decoder that estimates the initial flow of uncoded data. The data rate of the system can be tuned (in order to optimally exploit the quality of the radio link) by the choice of the data symbol constellations and the rate of the encoder. The available constellations for the mapping of binary data into data symbols are Gray-coded BPSK, QPSK, 16-QAM and 64-QAM (respectively 1, 2, 4 and 6 bits per data symbol). Depending on the chosen constellation, the standard allows to pick rate $1 / 2,3 / 4$ or $2 / 3$ codes (rate $r=2 / 3$ and $r=3 / 4$ codes result from puncturing a unique rate $r=1 / 2$ mother code). The allowed combinations of constellations and coding rates are detailed in Table 1 together with the resulting bit rates.

The achievable transmission speed for a target BER as a function of the SNR is plotted in Fig. 1. For the sake of clarity, only the best performing schemes are included in the figures. Uncoded OFDM is not shown, neither is rate 3/4 coding for KSP transmission. The results are shown in Fig. 1(a) for a target BER of $10^{-3}$ and in Fig. 1(b) for a target BER of $10^{-4}$. Uncoded KSP dominates the other techniques in the high SNR region. Rate $1 / 2$ coded KSP dominates in the low SNR region. KSP with FD equalization offers a similar performance 
Table 1 Data rate obtained with different constellations and coding rates

\begin{tabular}{lll}
\hline Data Rate $($ Mbits/s) & Modulation & Coding Rate \\
\hline 6 & BPSK & $1 / 2$ \\
9 & BPSK & $3 / 4$ \\
12 & QPSK & $1 / 2$ \\
18 & QPSK & $3 / 4$ \\
24 & 16 QAM & $1 / 2$ \\
36 & 16 QAM & $3 / 4$ \\
48 & 64 QAM & $2 / 3$ \\
54 & 64 QAM & $3 / 4$ \\
\hline
\end{tabular}
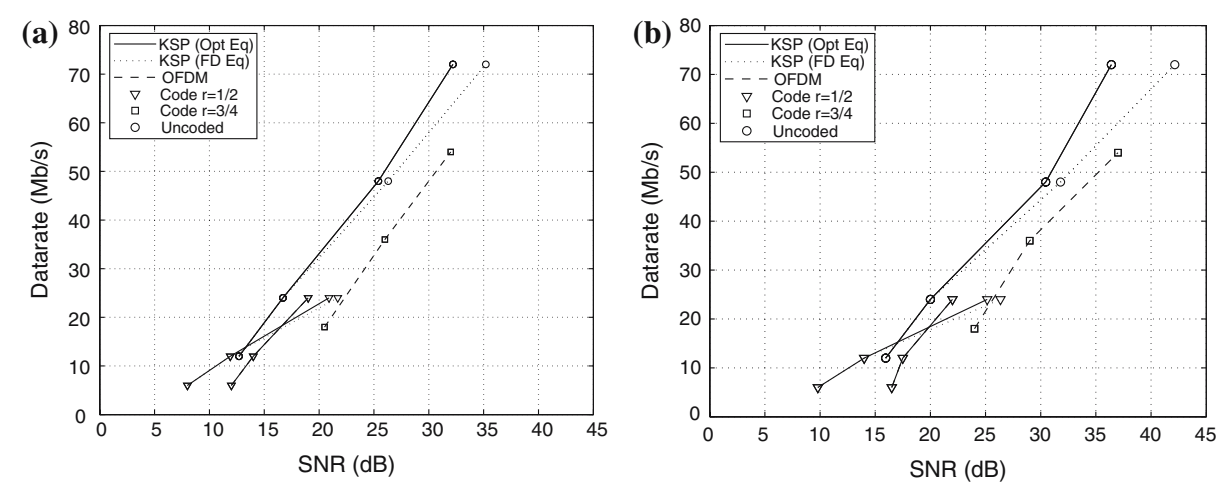

Fig. 1 Achievable data rates (Mbits/s) as a function of the SNR for a target BER of $10^{-3}$ (a) and $10^{-4}(\mathbf{b})$ for different transmission schemes

as KSP with optimal equalization for most SNRs, except for the highest SNRs. Both KSP schemes always outperform OFDM.

The good performance of uncoded KSP transmission makes it a suitable transmission scheme in the presented idealized WLAN context (i.e., no interferences, no burst noise, perfect time and frequency synchronization,...). The advantage of uncoded KSP transmission is twofold: the absence of coding does not only save the computationally demanding steps of coding and Viterbi decoding, it also avoids the redundant symbols introduced by the codes and thereby increases the throughput of the system. But coding could still be important to combat for instance interferences, burst noise, or synchronization errors.

\subsection{System Performance with Realistic Channel Estimates}

In this section, we consider the more realistic situation where the receiver first estimates the channel and then computes the equalizers relying on the estimated channel impulse response. The channel estimates for CP systems rely on two blocks of training symbols inserted at the beginning of each data burst as proposed in the IEEE 802.11a standard. For KSP systems, no training symbols are inserted and the channel estimate relies solely on the knowledge of the padded sequences. The channel estimates are obtained from the Gaussian ML method proposed in [8] considering 50 blocks of data symbols. We consider short padded sequences 

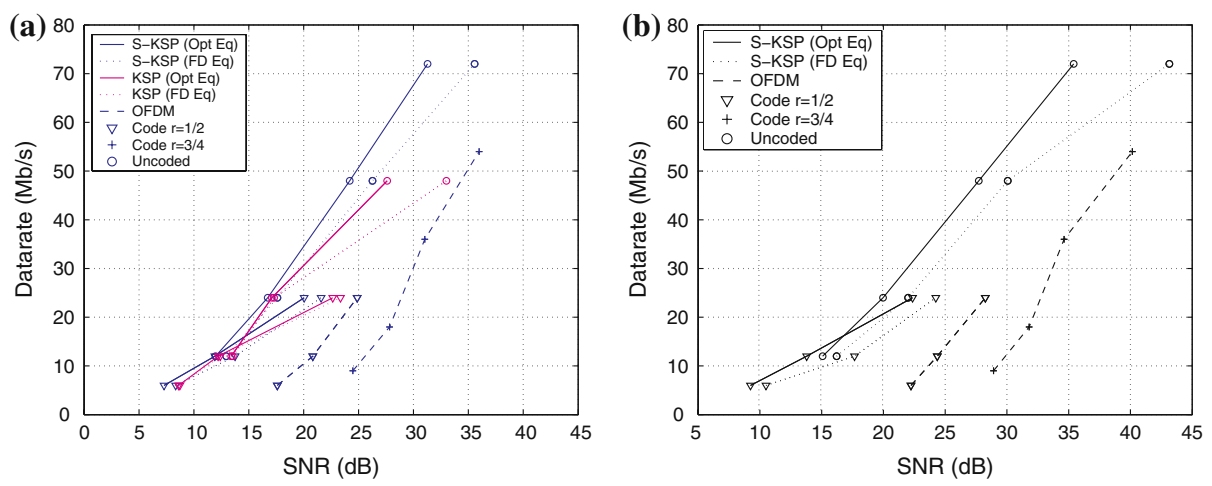

Fig. 2 Achievable data rates (Mbits/s) as a function of the SNR for a target BER of $10^{-3}$ (a) and $10^{-4}$ (b) for different transmission schemes when realistic channel estimates are considered

( $N_{t}=8$, which corresponds to the optional short CP length). S-KSP as well as classical $\mathrm{KSP}$ with constant padded sequences is considered. Perfect knowledge of the noise power is assumed for the design of the LMMSE equalizers. Perfect time and frequency synchronization is assumed as well.

In Fig. 2, we depict the performance of the different block transmission techniques when realistic channel estimates are considered. The achievable data rate is presented as a function of the SNR for a fixed target BER. As before, Fig. 2(a) considers a target BER of $10^{-3}$ and Fig. 1(b) considers a target BER of $10^{-4}$. The plots show that S-KSP outperforms both OFDM and classical KSP with constant padded sequences. The poor channel estimation performance of OFDM and classical KSP with constant padded sequences is highlighted by these experiments, since performance is significantly degraded compared to the perfect CSI case. Classical KSP with constant padded sequences is not even displayed in Fig. 2(b) as it fails to achieve the target BER of $10^{-4}$ for most constellations and coding rates.

\section{Conclusions}

In this paper, we have proposed a new block transmission technique, namely shifted KSP (S-KSP), which simultaneously allows for low-complexity frequency-domain equalization and accurate channel estimation. The performance of this scheme has been compared with other block transmission schemes in the light of the WLAN standard IEEE802.11a. From the presented perspective, the proposed S-KSP scheme outperforms other block transmission techniques and performs clearly better than OFDM as adopted in the standard. The KSP scheme, and more in particular S-KSP, then offers five major advantages over OFDM. The first advantage is that S-KSP has a reduced peak to average power ratio (PAPR) compared to OFDM. The second advantage results from the implicit encoding of the transmitted information over the tones. The third advantage resides in the fact that although both S-KSP and OFDM allow for the use of computationally efficient frequency-domain equalizers, the S-KSP performance can be further improved at the cost of an increased equalization complexity when the optimal KSP equalizers are used. The fourth advantage includes the possibility of estimating the channel accurately solely relying on the knowledge of the padded sequences that replace the classical CP. This allows to avoid the insertion of long preambles used for 
channel estimation whilst allowing for more accurate channel estimates. Similarly, the padded sequences can be exploited for time and frequency synchronization. Finally, the fifth advantage is that S-KSP offers the possibility of accurately tracking the evolution of timevarying channels (as well as time and frequency offsets) in mobile environments, because the training is present in every block [11].

Acknowledgement This research work was carried out at the ESAT laboratory of the Katholieke Universiteit Leuven, in the frame of the Belgian Program on Interuniversity Attraction Poles, initiated by the Belgian Federal Science Policy Office IUAP P5/11 ('Mobile multimedia communication systems and networks'). Geert Leus is supported in part by the NWO-STW under the VIDI program (DTC.6577).

\section{References}

1. Bingham, J. A. C. (1990). Multicarrier modulation for data transmission: An idea whose time has come. IEEE Communications Magazine, 5-14. May 1990.

2. Sari, H., Karam, G., \& Jeanclaude, I. (1995). Transmission techniques for digital terrestrial TV broadcasting. IEEE Communicatons Magazine, 100-109. Feb. 1995.

3. Wang, Z., \& Giannakis, G. B. (2000). Wireless multicarrier communications: Where fourier meets shannon. IEEE Signal Processing Magazine, 29-48. May 2000.

4. Deneire, L., Gyselinckx, B., \& Engels, M. (2001). Training sequence vs. Cyclic prefix: A new look on single carrier communication. IEEE Communication Letters, 5(7), 292-294.

5. Stoica, P., \& Besson, O. (2003). Training sequence design for frequency offset and frequency-selective channel estimation. IEEE Transactions on Communications, 51(11), 1910-1917.

6. Ciblat, P., \& Vandendorpe, L. (2002). On the maximum-likelihood based data-aided joint frequency offset and channel estimates. In Proceedings of the European signal Processing Conference (EUSIPCO 2002), Toulouse, France, Sept. 2002.

7. Leus, G., \& Moonen, M. (2001). Semi-blind channel estimation for block transmission with non-zero padding. In Proceedings of the Asilomar Conference on Signals, Systems and Computers, Pacific Grove, California, Nov. 2001.

8. Rousseaux, O., Leus, G., Stoica, P., \& Moonen, M. (2005). Gaussian maximum likelihood channel estimation with short training sequences. IEEE Transactions on Wireless Communications, 4(6), 2945-2955.

9. Cendrillon, R., \& Moonen, M. (2001). Efficient equalizers for single and multi-carrier environments with known symbol padding. In Proceedings of the International Symposium on Signal Processing and its Applications (ISSPA 2001), Kuala-Lumpur, Malaysia, Aug. 2001.

10. Manton, J. H., \& Hua, Y. (2000). Affine precoders for reliable communications. In Proceedings of the International Conference on Acoustics, Speech, and Signal Processing (ICASSP 2000), Istanbul, Turkey, June 2000.

11. Rousseaux, O., Leus, G., \& Moonen, M. (2006). Estimation and equalization of doubly selective channels using known symbol padding. IEEE Transactions on Signal Processing, 54(3), 979-990.

\section{Author Biographies}

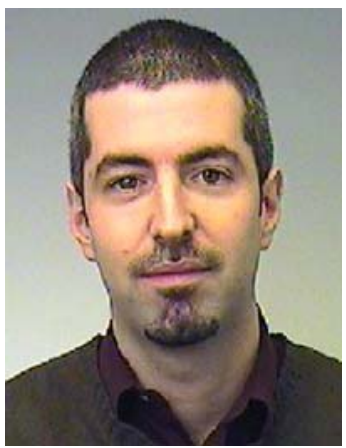

Olivier Rousseaux is Activity Leader and Senior Researcher for the Wireless group of IMEC-NL; his current research activities focuses on air interface design, system architecture, algorithmic and baseband solutions for Ultra Low Power Radios, with a more specific focus on low rate UWB IR radios. He previously worked as a researcher for the Katholieke Universiteit Leuven, with a specific focus on Block Transmission Techniques for High Data Rate Wireless Communications in a mobile environment. He received his M.Sc degree in Electrical and Mechanical Engineering from the Universite Catholique de Louvain and received his $\mathrm{PhD}$ degree from the Katholieke Universiteit Leuven with key expertise on Signal Processing for Wireless Communications. 


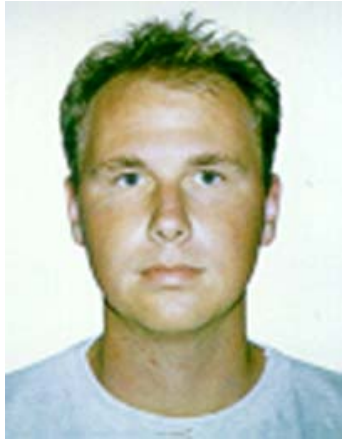

Geert Leus was born in Leuven, Belgium, in 1973. He received the electrical engineering degree and the $\mathrm{PhD}$ degree in applied sciences from the Katholieke Universiteit Leuven, Belgium, in June 1996 and May 2000, respectively. He has been a Research Assistant and a Postdoctoral Fellow of the Fund for Scientific Research - Flanders, Belgium, from October 1996 till September 2003. During that period, Geert Leus was affiliated with the Electrical Engineering Department of the Katholieke Universiteit Leuven, Belgium. Currently, Geert Leus is an Assistant Professor at the Faculty of Electrical Engineering, Mathematics and Computer Science of the Delft University of Technology, The Netherlands. During the summer of 1998, he visited Stanford University, and from March 2001 till May 2002 he was a Visiting Researcher and Lecturer at the University of Minnesota. His research interests are in the area of signal processing for communications. Geert Leus received a 2002 IEEE Signal Processing Society Young Author Best Paper Award and a 2005 IEEE Signal Processing Society Best Paper Award. He is a member of the IEEE Signal Processing for Communications Technical Committee, and an Associate Editor for the IEEE Transactions on Signal Processing and the EURASIP Journal on Applied Signal Processing. In the past, he has served on the Editorial Board of the IEEE Signal Processing Letters and the IEEE Transactions on Wireless Communications.

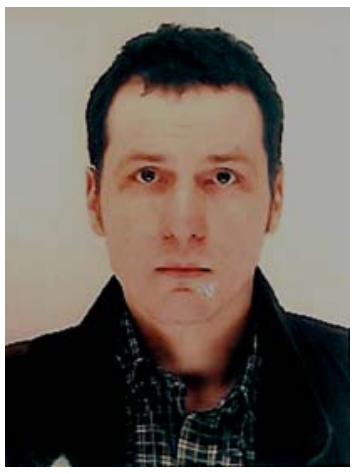

Marc Moonen received the electrical engineering degree and the Ph.D. degree in applied sciences from Katholieke Universiteit Leuven (K.U. Leuven), Belgium, in 1986 and 1990, respectively. Since 2004, he has been a Full Professor at the Electrical Engineering Department of Katholieke Universiteit Leuven, where he is currently heading a research team of $16 \mathrm{Ph} . \mathrm{D}$. candidates and postdocs, working in the area of numerical algorithms and signal processing for digital communications, wireless communications, DSL, and audio signal processing. Dr. Moonen received the 1994 K.U. Leuven Research Council Award, the 1997 Alcatel Bell (Belgium) Award (with Piet Vandaele), and the 2004 Alcatel Bell (Belgium) Award (with Raphael Cendrillon) and was a 1997 Laureate of the Belgium Royal Academy of Science. He received a journal Best Paper Award (with Geert Leus) from the IEEE Transactions on Signal Processing and (with Simon Doclo) from Elsevier Signal Processing. He was Chairman of the IEEE Benelux Signal Processing Chapter from 1998 to 2002 and has been a European Association for Signal, Speech and Image Processing (EURASIP) AdCom Member since 2000 and a member of the IEEE Signal Processing Society Technical Committee on Signal Processing for Communications. He has served as Editor-in-Chief for the EURASIP Journal on Applied Signal Processing from 2003 to 2005 and has been a member of the editorial board of IEEE Transactions on Circuits and Systems I from 2002 to 2003 and IEEE Signal Processing Magazine from 2003 to 2005 . He is currently a member of the editorial board of Integration, the VLSI Journal, EURASIP Journal on Applied Signal Processing, EURASIP Journal on Wireless Communications and Networking, and Signal Processing. 